$\mathrm{M} / \mathrm{C}-\mathrm{TH} 93 / 13$

RAL-93-030XRS:

\section{On the Interpretation of the HERA Total Cross Section Measurement}

\author{
J.R.Forshaw ${ }^{1}$ and J.K.Storrow ${ }^{2}$
}

1 Theoretical Physics Division, Rutherford Appleton Laboratory, Chilton, Didcot, Oxon. England.

${ }^{2}$ Department of Theoretical Physics, University of Manchester, Manchester, England.

\section{Abstract}

The implications for perturbative QCD minijet calculations of combining the recent HERA measurements of the total $\gamma p$ cross section with the lower energy data are discussed.

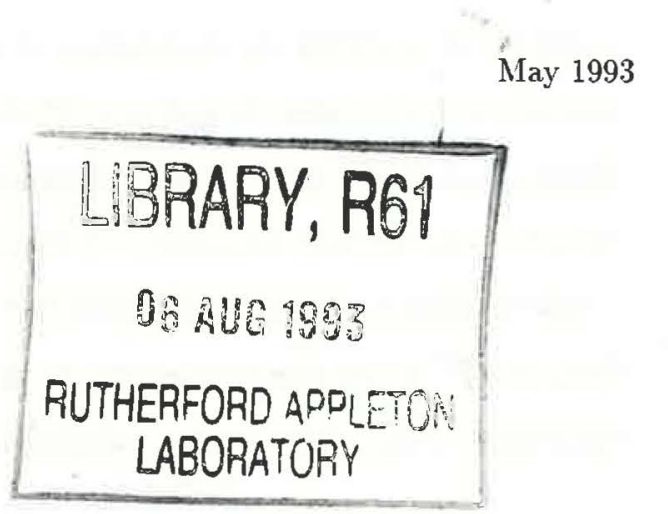

Recently both the H1 [1] and ZEUS [2] collaborations at the DESY ep collider HERA have published results for the total $\gamma p$ cross section at centre of mass energies of $\simeq 200 \mathrm{GeV}$. The $\mathrm{H} 1$ collaboration $(\langle\sqrt{ } s\rangle=195 \mathrm{GeV})$ find:

$$
\sigma_{T}^{\gamma p}=(159 \pm 7 \pm 20) \mu \mathrm{b}
$$

and $\operatorname{ZEUS}(\langle\sqrt{ } s\rangle=210 \mathrm{GeV})$ obtain

$$
\sigma_{T}^{\gamma p}=(154 \pm 16 \pm 32) \mu \mathrm{b}
$$

In each case, the first error is statistical and the second systematic. In this short note we wish to discuss these numbers in the context of the perturbative QCD (pQCD) minijet model calculations which have been published [3-6].

We will assume that the total cross section can be represented as the sum of two components: a soft (non-perturbative) component and a hard (perturbative) component, i.e.

$$
\sigma_{T}^{\gamma p}(s)=\sigma_{T}^{s o f t}(s)+\sigma_{T}^{p Q C D}(s)
$$

This is a very crude ansatz and it may well be that there is no simple way of disentangling the soft and hard components to the total cross section. The ansatz provides us with a first approximation, and does allow some definite conclusions to be drawn.

Starting from the inclusive 2-jet cross section calculated using $\mathrm{PQCD}$, and realising that multiple parton scattering may well be important at HERA energies, the following eikonal formula for the minijet contribution to the total cross section has been advocated:

$$
\sigma_{T}^{p Q C D}=P_{h a d} \int_{0}^{\infty} \pi d b^{2}[1-\exp (-\chi(b, s))] .
$$

The eikonal, $\chi(b, s)$, is assumed to be of the form:

$$
\chi(b, s)=A(b) \sigma_{\text {hard }}(s) / P_{\text {had }} .
$$


The hard scattering cross section is just the 2-jet inclusive cross section integrated over all $p_{T} \geq p_{T \text { min }}$, where $p_{T \text { min }}$ is a necessary delineating scale below which the use of $\mathrm{pQCD}$ cannot be justified, and the physics below $p_{T \text { min }}$ is then assumed to be contained in $\sigma_{T}^{\text {soft }}$. The factor $A(b)$ describes the overlap of partons within the colliding particles (photon and proton) in impact parameter space and is usually taken to be a convolution of the electromagnetic form factors. The factor $P_{\text {had }}$ is the probability that the photon interacts like a vector meson, which is expected to be $\sim 4 \pi \alpha_{e m} / f_{\rho}^{2}(\simeq 1 / 300)[4-8]$. Manifest in eqn.(4) is the possibility of more than one hard scatter per $\gamma p$ interaction. The eikonal is interpreted as the mean number density of hard scatters for collisions at impact parameter $b$, and hence $\exp (-\chi(b, s))$ is the probability of no hard scatter at impact parameter $b$. The explicit form for $\sigma_{\text {hard }}$ is:

$$
\sigma_{\text {hard }}(s)=\sum_{i, j, k, l} \int_{p_{T \min }^{2}}^{s / 4} d p_{T}^{2} \int_{4 p_{T}^{2} / s}^{1} d x_{1} \int_{4 p_{T}^{2} x_{1} / s}^{1} d x_{2} f_{i / \gamma}\left(x_{1}, Q^{2}\right) f_{j / \gamma}\left(x_{2}, Q^{2}\right) \frac{d \sigma_{i j \rightarrow k l}}{d p_{T}^{2}}(s)
$$

where $Q^{2}$ is the QCD scale, usually taken to be given by the hard scattering scale $Q^{2} \sim p_{T}^{2}$. Recalling that an inclusive cross section integrated over $p_{T}$ gives a total cross section multiplied by a multiplicity (here it is the multiplicity of hard scatters), then the eikonal prescription gives a simple model for this multiplicity, based on the distribution of events in impact parameter space and the size of the cross section. This is a minimal eikonal prescription, which is specifically designed to handle the multiplicity of hard scatters and is in contrast with other models which use an eikonal which is a sum of both hard and soft components. The two approaches are essentially equivalent in the absence of any theoretical handle on the soft physics.

Let us briefly discuss the approximations and uncertainties inherent in the eikonal formalism (for a more complete discussion, we refer to refs. $[4,5]$ ). Poissonian statistics are behind the formalism, in particular one assumes that separate hard scatters are incoherent and that the parton model is valid. The assumption that one can treat the photon using the vector meson dominance model (VMD) leads to the factorisation of the $P_{\text {had }}$ factor. This is a reasonable assumption since any contributions to the total cross section from the so-called anomalous and direct components are expected to be small [7]. As discussed by Honjo et al [8], one can expect higher-mass vector meson states (i.e. those beyond the simple VMD contribution) to be present, and a more complicated eikonalisation formula is required $[7,8]$. However we expect the essential features to be contained in the simple formulae above with a judicious choice of $P_{\text {had }}$ and $p_{\text {Tmin }}$.

Even assuming the eikonal formula, there are a number of additional uncertainties. The most important of these is the choice of $p_{T \text { min }}$ and the related question of the ansatz used to determine the soft cross section. For example, one could take a large enough value of $p_{\text {Tmin }}$ such that the contribution from the hard component is negligible and the whole of the cross section must be attributed to $\sigma_{T}^{\text {soft }}$. For the minijet approach to be interesting at HERA energies (i.e. for hard scattering to contribute significantly to the total cross section), one relies upon the validity of pQCD down to $p_{T \text { min }} \simeq 2-3 \mathrm{GeV}$. The soft component is often chosen to ensure a constant total cross section in the absence of any hard scattering component. This is merely an ansatz which has very little theoretical motivation beyond the cosmetic attraction of providing an 'explanation' of the rise in total cross sections. Such an assumption is equivalent to making a rather ad hoc separation of the hard and soft physics, and endows $p_{T \min }$ with great physical significance as the scale which delineates the hard and soft components of the pomeron. It is far from clear that such a simple separation is appropriate and in fact we will be forced by the data to take a form for $\sigma_{T}^{s o f t}$ which rises with energy. In fig. 1 we show the results of ref.[5] for the eikonalised minijet cross section, calculated with $p_{T \min }=3 \mathrm{GeV}$, after adding the 
following cross section for the soft physics:

$$
\sigma_{T}^{s o f t}(s)=A s^{e}+\frac{B}{\sqrt{ } s} .
$$

The choice $A=78.4 \mu \mathrm{b}, \epsilon=0.058$ and $B=117.05 \mu \mathrm{b} \mathrm{GeV}$, ensures an excellent fit to the low energy data (i.e. $\sqrt{ } s \lesssim 18 \mathrm{GeV}$ ) [9]. We note particularly the fact that the data in the 10-18 GeV range shows evidence of a rise with energy, a fact that is usually ignored in minijet studies, despite being known for many years. If one were to attribute this low energy rise to the minijet contribution then one would be forced to choose very low values of $p_{T \text { min }}$, in the $1-1.5 \mathrm{GeV}$ range, which would lead to much too high a prediction at HERA. This is illustrated by the second curve shown in fig.1, where we add to the minijet cross section of ref.[5], calculated with $p_{\text {Tmin }}=2 \mathrm{GeV}$, the soft cross section:

$$
\sigma_{T}^{s o f t}(s)=A+\frac{B}{\sqrt{ } s}
$$

with $A=107.98 \mu \mathrm{b}$ and $B=54.34 \mu \mathrm{b} \mathrm{GeV}$. This leads to an unconvincing description of the low energy rise and predicts quite a high cross section at HERA energies. Any reduction of $p_{T \min }$ to improve the former feature would lead to a worsening of the latter.

In attributing only part of the rising cross sections to minijets we are taking a slightly unorthodox stance, but we would argue that this is a more rational view of the situation. We emphasize that the minijet question is not a stark choice between the two extremes of either minijets providing the entire rise with energy of the total cross section or being absent: indeed they have been shown to be present in both $\bar{p} p$ [10] and $\gamma \gamma[11]$ reactions. The important issue is whether they make a significant contribution to the total cross section at existing energies. In this scenario with $p_{T \text { min }}=3 \mathrm{GeV}$ they do, $\sigma_{T}^{p Q C D}$ being around $20 \mu \mathrm{b}$ at $\sqrt{ } s=200 \mathrm{GeV}$. To emphasize the sensitivity of $\sigma_{T}^{p Q C D}$ to $p_{T \min }$ mentioned earlier we note that with $p_{T_{\min }}=2 \mathrm{GeV}$ the corresponding cross section is $70 \mu \mathrm{b}$.

Additional uncertainties, which are far from negligible, arise from the leading order nature of the calculation. They come from the choice of the QCD scale $Q^{2} \sim p_{T}^{2}$ in eq.(6), and from the possible effects of higher order QCD corrections. Both can be expected to be significant, particularly the latter since the dominant contribution arises from minijets produced at rather low $p_{T}$ and so the relevant $\alpha_{s}\left(Q^{2}\right)$ will be relatively large. Over and above all of this is the uncertainty due to the lack of knowledge of the parton distributions of the photon, particularly of the gluon at small $x$.

It is the cumulative effect of all these uncertainties which means that a single measurement of the total cross section is unlikely to tell us much, e.g. which of the presently available parton distributions are more reliable. One can however conclude that the more extreme predictions (for the total cross section) which have been made in the past are incorrect. Specifically, the HERA measurements do not support the very rapidly rising total cross section predicted in earlier calculations which did not include the effects of multiple scattering and used a very steep small $x$ gluon component of the photon [3]. We consequently agree with the conclusion of the H1 collaboration (who used PYTHIA without multiple scattering to compare with the data), that minijet calculations with $p_{T \text { min }} \lesssim 1.4 \mathrm{GeV}$ seem to be ruled out, i.e. only with unrealistically small values of $P_{\text {had }}$ and/or $\sigma_{T}^{\text {soft }}$ could such predictions agree with the HERA data. We point out that although it would appear that more data points and smaller errors would be able to distinguish between different models (e.g. the Donnachie-Landshoff model [12] and the minijet model) that in fact this is not the case. The aforementioned uncertainties inherent in the calculations would make it almost impossible to rule out the minijet model through a study 
of the total cross section alone, though it would be helpful to have measurements at different energies. Similarly, one cannot expect to unambiguously observe the effects of multiple scattering at HERA through a study of the total cross section. It is therefore necessary to confront the minijet model with data on the structure of the final state before any conclusions can be made on its validity. The most efficient way of performing such a comparison is through a Monte Carlo and the recent work by Schuler and Sjöstrand goes some way towards this goal [13].

Consequently, any claim to make definite predictions for the $\gamma p$ total cross section using minijets must be misleading. The recent work by Fletcher et al [6] proposes using the Tevatron data and the additive quark model to fix $p_{T \min }$ and the soft cross section. A canonical low energy value for the $\gamma p$ cross section is then used to fix $P_{\text {had }}$ [6] - i.e. no attempt is made to fit the energy dependence of the low energy data. This is a valid endeavour but cannot claim to make definite predictions, serving only to show how a sensible minijet model can lead to results which are consistent with the present HERA data. For example, any future deviation from the predictions of ref.[6] would not be evidence for the failure of the minijet model. This approach is to contrasted to that of of Donnachie and Landshoff [12], which is a specific extrapolation of a fit to the low energy data using a parametrisation motivated by analyses of the total cross section data on many reactions, and so has much less flexibility.

In summary therefore, we hope to have clarified the situation with regards to minijets in the light of the recent HERA data. Looking only at the total cross section will not establish the validity or otherwise of current (i.e. leading order) minijet models, and a more detailed Monte Carlo study of the final state is necessary to meet this goal. The present data do however have something to say with respect to minijets: the more extreme predictions for $\sigma_{T}^{\gamma p}$ are ruled out, and consequently so are very small values of $p_{T \min }$ (i.e. as low as $1 \mathrm{GeV}$ ). Our analysis also provides indications that not all of the rise in total cross sections should be ascribed to minijets. 


\section{References}

[1] T.Ahmed et al (H1 Collaboration), Phys.Lett. B299, 374 (1993).

[2] M.Derrick et al (ZEUS Collaboration), Phys.Lett. B293, 465 (1992).

[3] M.Drees and F.Halzen, Phys.Rev.Lett. 61, 275 (1988); R.Gandhi and I.Sarcevic, Phys.Rev. D44, R10 (1991).

[4] J.C.Collins and G.A.Ladinsky, Phys.Rev. D43, 2847 (1991); J.R.Forshaw and J.K.Storrow, Phys.Lett. B268, 116 (1991); erratum B276, 565 (1992); R.S.Fletcher, T.K.Gaisser and F.Halzen, Phys.Rev D45, 337 (1992); erratum D45, 3279 (1992).

[5] J.R.Forshaw and J.K.Storrow, Phys.Rev. D46, 4955 (1992).

[6] R.S.Fletcher, T.K.Gaisser and F.Halzen, Phys.Lett. B298 (1993) 442.

[7] J.R.Forshaw, Phys.Lett. B285, 354 (1992)

[8] K.Honjo et al, 'High Energy Photon-Nucleon and Photon-Nucleus Cross Sections', Madison Preprint 1992.

[9] D.O.Caldwell et al, Phys.Rev. D7, 1362 (1973); Phys.Rev.Lett. 40, 1222 (1978).

[10] C.Albajar et al (UA1 Collaboration), Nucl.Phys. B309, 405 (1988).

[11] R.Tanaka et al (AMY Collaboration), Phys.Lett. B277, 215 (1992).

[12] A.Donnachie and P.V.Landshoff, Nucl.Phys. B231, 189 (1984); Phys.Lett B296 227 (1992).

[13] G.A.Schuler and T.Sjöstrand, Phys.Lett. B300 169 (1993); 'Towards a Complete Description of High-Energy Photoproduction', CERN-TH.6796/93.
[14] J.Morfin and Wu-Ki Tung, Z.Phys. C52, 13 (1991).

[15] M.Drees and K.Grassie, Z.Phys. C28, 451 (1985). 


\section{FIGURE CAPTION}

Figure 1 : Plot of $\sigma_{T}^{\gamma p}$ against $\sqrt{ } s$. Solid line: $\sigma_{T}^{\text {soft }}$ of eqn.(7) and $\sigma_{T}^{p Q C D}$ with $\ddot{p}_{T \min }=3 \mathrm{GeV}$. Broken line: $\sigma_{T}^{\text {soft }}$ of eqn. $(8)$ and $\sigma_{T}^{p Q C D}$ with $p_{T \text { min }}=2 \mathrm{GeV}$. The data are from refs.[1, 2, 9] and the structure functions of ref.[14, 15] were used when computing $\sigma_{T}^{p Q C D}$.

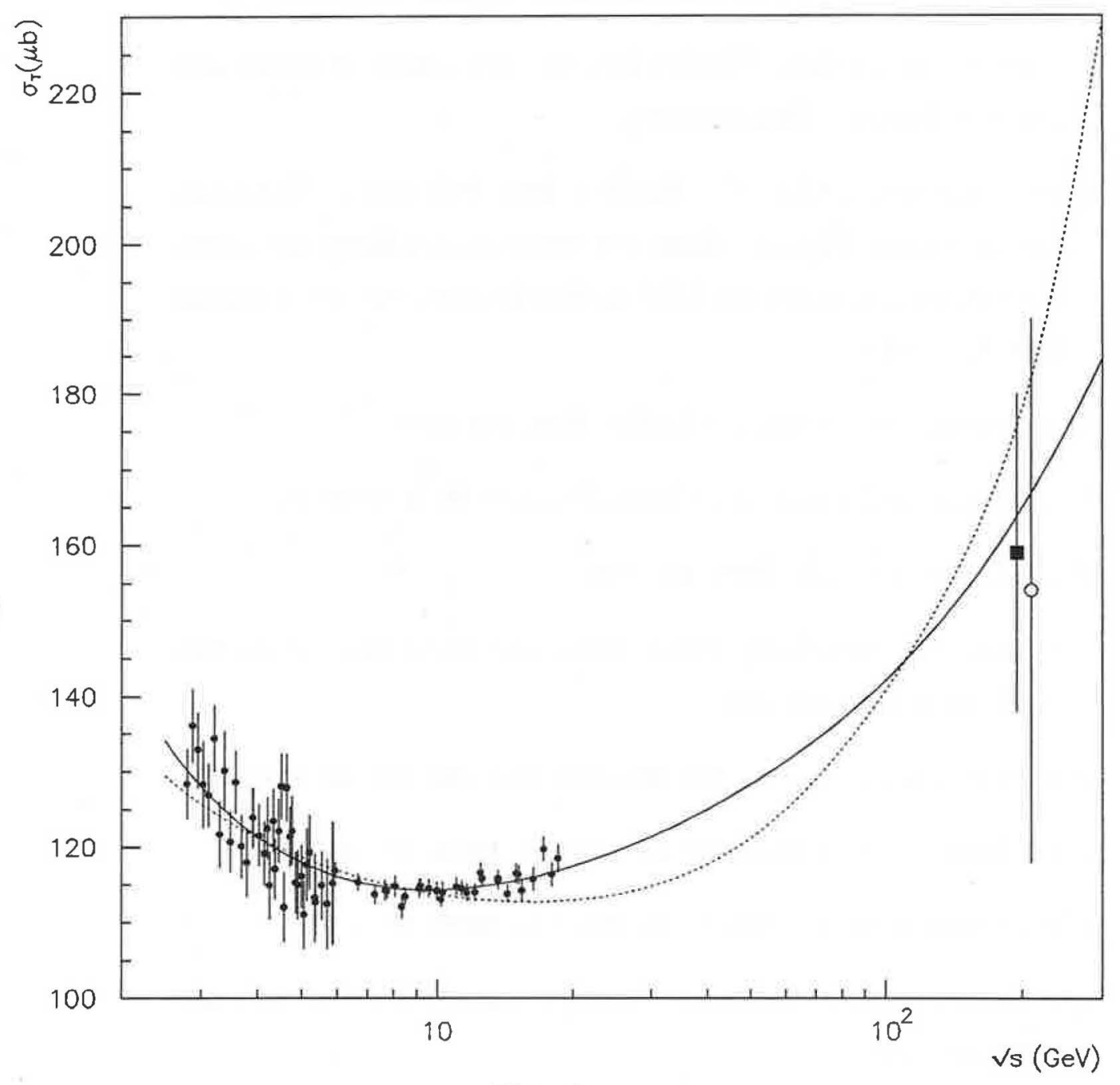

Fig. 1 